\title{
Meta
}

Journal des traducteurs

Translators' Journal

\section{Le rôle de la terminologie et de la documentation dans l'enseignement de la traduction spécialisée}

\section{Geneviève Mareschal}

Volume 33, numéro 2, juin 1988

L'enseignement de la traduction au Canada - Teaching Translation in Canada

URI : https://id.erudit.org/iderudit/003573ar

DOI : https://doi.org/10.7202/003573ar

Aller au sommaire du numéro

Éditeur(s)

Les Presses de l'Université de Montréal

ISSN

0026-0452 (imprimé)

1492-1421 (numérique)

Découvrir la revue

Citer cet article

Mareschal, G. (1988). Le rôle de la terminologie et de la documentation dans l'enseignement de la traduction spécialisée. Meta, 33(2), 258-266.

https://doi.org/10.7202/003573ar d'utilisation que vous pouvez consulter en ligne. 


\section{LE RÔLE DE LA TERMINOLOGIE ET DE LA DOCUMENTATION DANS L'ENSEIGNEMENT DE LA TRADUCTION SPÉCIALISÉE}

GENEVIÈve MARESCHAL

Universitê d'Ottawa, Ottawa, Ontario

Avant d'aborder le rôle joué par la terminologie et la documentation dans l'enseignement de la traduction spécialisée, il convient de définir ce que nous entendons par traduction spécialisée. Lorsqu'on jette un coup d'œil aux contenus des programmes de traduction des universités, on constate que ce syntagme figure généralement au tableau des matières enseignées au futur traducteur, au même titre que la traduction générale et la traduction technique. Et lorsqu'on analyse la description des cours de traduction spécialisée dans les annuaires universitaires, on apprend que la traduction spécialisée consiste en la traduction de textes administratifs, commerciaux, juridiques, sociologiques, économiques, politiques ou autres ${ }^{1}$. Par opposition à la traduction spécialisée, la traduction technique est généralement définie comme la traduction de textes se rapportant à des domaines techniques et scientifiques. Quant à la traduction générale, souvent maladroitement définie d'ailleurs ${ }^{2}$, elle regroupe un éventail de sujets avec pour communs dénominateurs le grand public comme destinataire, la langue écrite contemporaine comme source et l'apprentissage de la traduction professionnelle comme objectif.

On peut certes contester la validité d'une "typologie " de ce genre ${ }^{3}$ en faisant valoir, par exemple, le manque de cohérence des critères de classement, mais notre propos n'est pas d'entrer dans une discussion philosophique de la raison d'être de catégories communément établies et reconnues au niveau de la formation universitaire. Pour les besoins de cet exposé, nous définirons traduction spécialisée comme la traduction de textes spécialisés, c'est-à-dire relevant d'un domaine de spécialisation ou d'une branche d'activité particulière et, à ce titre, tout texte portant sur un domaine précis de la connaissance humaine doit être considéré comme spécialisé.

Or, de quoi se compose un texte spécialisé ? Qu'est-ce qui le distingue d'un texte non spécialisé ? D'où tire-t-il sa spécificité ? Un texte spécialisé traite d'un sujet précis, à l'intérieur d'un champ de spécialisation donné, et se caractérise par un vocabulaire et une phraséologie propres. La traduction d'un texte spécialisé comporte donc deux dimensions essentielles : d'une part, l'objet du texte ou son contenu et, d'autre part, la langue du texte ou sa forme. Un texte économique differe, dans son contenu et dans sa forme, d'un texte juridique ou d'un texte médical. C'est ce contenu et cette forme que le traducteur est appelé à transférer d'une langue $\mathrm{A}$ vers une langue $\mathrm{B}$, en en respectant tous les éléments constituants. Et l'objectif d'un cours de traduction spécialisée est précisément d'apprendre à l'étudiant à effectuer ce transfert, dans les règles de l'art.

Quelle est la situation de l'étudiant face à un texte spécialisé ? En règle générale, il se trouve dans une situation d'incompétence. Incompétence à la foís notionnelle et linguistique. L'étudiant type, c'est-à-dire celui qui vient de terminer ses études secondaires et qui s'engage directement dans la voie universitaire, n'a du monde qui l'entoure 
qu'une connaissance et une expérience réduites et souvent théoriques. Il dispose évidemment d'un bagage culturel, scientifique et technique élémentaire, mais il n'a pas eu le temps ni l'occasion de parfaire ses connaissances ni de les mettre à l'épreuve de la réalité quotidienne. Sur le plan linguistique, sa formation générale lui aura permis d'acquérir la langue usuelle et peut-être aussi les éléments de base de la langue scientifique ${ }^{4}$, mais on ne peut s'attendre qu'il ait véritablement été initié à ce qu'il est convenu d'appeler une langue de spécialités. Et quand bien même il aurait acquis une spécialisation et la langue de spécialité s'y rapportant, il ne s'agirait encore que d'un domaine parmi beaucoup d'autres. En fait, l'étudiant du cours de traduction spécialisée se trouve dans la même situation que le traducteur professionnel qui se voit confier un texte relevant d'un domaine qui lui est totalement étranger. Le traducteur, tout comme l'étudiant, se trouve alors dans une situation d'incompétence. Certes, son expérience de la traduction, sa nneilleure connaissance de la réalité quotidienne et sa plus grand maturité lui permettront de résoudre plus aisément que l'étudiant les problèmes que le texte posera, mais il n'en demeure pas moins qu'il lui faudra acquérir les concepts et la langue du domaine inconnu pour pouvoir effectuer une traduction de qualité.

Le cours de traduction spécialisée devra donc viser ce double objectif, notionnel et linguistique. Avec l'aide du professeur, l'étudiant doit apprendre à se documenter rapidement et efficacement sur un sujet spécialisé et à utiliser de façon appropriée les nouvelles connaissances acquises. Cette recherche tant documentaire que terminologique suppose en fait l'acquisition et la mise en pratique d'une méthode de travail. Cette méthode devra permettre à l'étudiant de s'approprier par lui-même les notions, la terminologie et la phraséologie dont il aura besoin pour traduire les textes spécialisés qui lui seront soumis, en classe d'abord, dans la profession ensuite. Nous ne saurions assez insister sur l'importance de ce par lui-même, car c'est un processus d'acquisition autonome qu'il convient de favoriser chez l'apprenti traducteur. Ce processus est, en effet, celui de la réalité professionnelle. Lorsqu'on confie un texte à un traducteur de métier, on attend de lui qu'il effectue la recherche documentaire et terminologique nécessaire. On lui fournit parfois une documentation de base, mais cette situation est loin d'être la règle et, dans la majorité des cas, le traducteur se trouve livré à lui-même. Il nous apparaît pédagogiquement important que la situation d'apprentissage reflète celle de la réalité.

La question qui se pose ici est de savoir comment la recherche documentaire et terminologique s'insère dans le processus traductionnel et quelle place il convient de lui consacrer dans l'enseignement de la traduction spécialisée. La réponse à cette question se fonde sur deux prémisses étroitement reliées entre elles. La première veut que ce soit le texte qui guide la recherche documentaire et terminologique et non le domaine de spécialisation auquel ce texte appartient. La seconde, qui découle directement de la première, pose que cette recherche est une recherche ponctuelle et non thématique, c'est-àdire portant sur "des termes isolés ou sur des groupes restreints de termes d'un même domaine $^{6}$ " et non "sur l'ensemble des termes appartenant à un domaine particulier ${ }^{7}$ ". Même si traduction et terminologie sont indissociables, le cours de traduction spécialisée n'est pas un cours de terminologie. Son objectif primordial est d'apprendre à traduire et, bien que cet apprentissage passe nécessairement par l'acquisition de notions et de leur dénomination, ces activités sont secondaires et subordonnées à l'opération de traduction. Dans la réalité du métier comme dans la formation, il n'est pas requis de celui qui traduit qu'il connaisse la terminologie du domaine ou du sous-domaine entier. C'est le sujet traité dans le texte qui servira à circonscrire l'étendue de la recherche terminologique à entreprendre.

Le processus traductionnel ${ }^{8}$ commence par une prise de connaissance globale du texte à traduire. Cette prise de connaissance peut aller de la lecture entière du texte, 
lorsqu'il n'est pas trop long ou qu'il s'agit d'une œuvre littéraire, jusqu'à un survol de la table des matières dans le cas d'un traité scientifique, en passant par la lecture partielle des différents chapitres d'un document imposant. Le degré d'approfondissement de cette première étape du travail du traducteur dépend essentiellement de la compétence de celui-ci, de la taille de document et de la nature du document. Dans le cas de l'étudiant, il va sans dire qu'une lecture complète s'impose, d'abord parce que les textes qui lui sont présentés sont généralement courts et ensuite, et surtout, parce qu'il n'a pas encore acquis l'expérience et la compétence requises pour "situer " un texte sur la base d'un simple coup d'œil. Cette lecture a pour but essentiel de permettre au traducteur de déterminer le sujet traité dans le document à traduire afin de pouvoir, dans un deuxième temps, sélectionner les outils d'information utiles à la compréhension du texte et à sa traduction. La détermination précise du sujet traité est primordiale : il faut que celui-ci soit cerné au plus près. Une bonne initiation aux méthodes de classement utilisées en bibliothéconomie et aux systèmes de classement des domaines des banques de terminologie faciliteront cette tâche ${ }^{9}$. Il faut que le traducteur sache que le droit, ou l'économie, ou la cybernétique se subdivisent en sous-domaines qui, eux-mêmes, se subdivisent en sous-catégories, etc., car il s'agit pour lui d'identifier avec précision, à partir du texte qui lui est soumis, le sous-domaine ou la sous-catégorie dont le texte relève. Inutile en effet de se documenter sur l'histoire du droit si le texte à traduire est un testament, ou de se procurer un dictionnaire technique général lorsqu'un lexique spécialisé en chaudronnerie conviendrait exactement au sujet traité. Non pas que l'histoire du droit ou le dictionnaire technique soient inutiles, mais parce que la formation à donner à un futur traducteur comporte une dimension opérationnelle. Les facteurs temps et rendement sont extrêmement importants sur le marché du travail et l'enseignant doit apprendre à l'étudiant à aller à l'essentiel et à ne pas se perdre en détails. Cette démarche est loin d'être facile et les traducteurs n'auront pas à fouiller bien loin dans leur mémoire pour se rappeler les tâtonnements de leurs débuts, les lectures inutiles, le temps perdu en compilations désordonnées.

Pour mieux illustrer la compétence documentaire et terminologique à donner à un étudiant dans un cours de traduction spécialisée, nous prendrons comme exemple la traduction d'un rapport annuel de l'anglais au français. Un rapport annuel se compose essentiellement de deux parties : une première partie, descriptive, qui fait le point sur les activités d'une entreprise au cours d'un exercice financier écoulé et sur ses perspectives d'avenir, et une deuxième partie, comptable, où se trouvent présentés les états financiers de l'entreprise. Il s'agit donc bien d'un texte spécialisé, qui porte d'ailleurs sur deux domaines d'activité particuliers et qui met en œuvre deux langues de spécialité. Dans son processus d'apprentissage, l'étudiant sera ainsi confronté à deux contenus et à deux discours différents, demandant des compétences documentaires et terminologiques différentes. De plus, c'est le genre de texte que tout traducteur risque de trouver un jour sur son bureau, et à ce titre, il a une valeur utilitaire certaine.

La compétence de recherche à acquérir et à mettre en application à l'étape de la prise de connaissance globale porte d'abord sur la délimitation précise du domaine de spécialité. Dans le cas d'un rapport annuel, l'étudiant devra en distinguer deux : l'un, commun à tous les rapports annuels, la comptabilité ; l'autre, variant d'une entreprise à l'autre en fonction des produits ou services offerts : pétrole, télécommunications, alimentation, etc.

Après l'étape de la prise de connaissance du texte et de la détermination du domaine vient l'étape de la sélection de la documentation, au cours de laquelle l'étudiant devra sélectionner les outils d'information qui lui permettront 1) de comprendre le texte à traduire et 2 ) de le traduire. Il s'agit donc pour lui de se procurer deux types d'ouvra- 
ges : paralexicographiques d'une part, c'est-à-dire d'initiation au sujet traité, et lexicographiques d'autre part, c'est-à-dire de traduction. Cette étape est beaucoup plus complexe et plus délicate qu'elle ne le parait de prime abord, principalement en raison de la "prolifération" documentaire contemporaine. Dans bien des domaines, l'étudiant risque en effet de se trouver submergé de documents, de qualité très variable toutefois. Or, à l'exception de quelques rares bibliographies annotées ou extrêmement sélectives, les bibliothèques et autres sources de documentation n'offrent généralement pas d'indication quant à la destination, l'utilité, la qualité et la fiabilité des ouvrages qu'elles proposent ou mettent à la disposition des utilisateurs. C'est donc le sens critique de l'étudiant qui sera sollicité et mis à l'épreuve pendant cette opération. En s'appuyant sur les techniques de recherche documentaire et sur les critères d'évaluation documentaire auxquels l'auront initié les cours de documentation et/ou de terminologie, l'étudiant apprendra à sélectionner les outils véritablement utiles et pertinents. Cet apprentissage, qui fait partie intégrante des objectifs d'un programme de traduction, est lent et difficile, et l'un des reproches les plus fréquemment formulés à l'endroit des traducteurs débutants, même sortis d'écoles de traduction, est qu'ils ne savent pas se documenter.

Sur le plan pédagogique, il importe donc que le professeur de traduction spécialisée favorise cet apprentissage en forçant l'étudiant à sélectionner lui-même les outils dont il aura besoin. Plutôt que de lui fournir une bibliographie spécialisée ou une liste de livres à consulter, le professeur demandera à chaque étudiant de lui soumettre un choix d'ouvrages et de justifier ce choix. Grâce à la connaissance qu'il a du domaine et de la documentation, le professeur procédera ensuite à l'analyse critique des ouvrages proposés et à la recommandation d'un ou deux ouvrages paralexicographiques de base et d'un nombre limité d'ouvrages lexicographiques, de façon à mieux former et à raffiner le sens critique de l'étudiant à l'égard de la documentation disponible.

La question qui se pose invariablement à ce stade-ci du processus traductionnel est de savoir si la documentation paralexicographique de base doit être choisie et lue en langue de départ ou en langue d'arrivée. Nous avons personnellement opté pour la langue d'arrivée, comme en témoigne le choix proposé plus loin. Le rôle des ouvrages de base est d'initier le traducteur à un sujet, c'est-à-dire de lui faire acquérir un bagage notionnel minimum dans le domaine traité, de façon à lui permettre de comprendre le texte de départ. Le rôle premier de ces ouvrages est donc informatif. À moins qu'aucune documentation ne soit disponible sur le sujet dans la langue d'arrivée, la lecture de ces documents en langue d'arrivée est plus rentable professionnellement, puisqu'elle permet d'atteindre deux buts à la fois : s'initier au domaine et prendre connaissance de la terminologie d'arrivée. Notion et terme étant indissociables, le traducteur, dont les sens linguistiques sont toujours en éveil, assimilera simultanément un certain nombre de notions et d'équivalents terminologiques.

Sur le plan lexicographique, la sélection effectuée par l'étudiant doit porter à la fois sur des dictionnaires bilingues et des dictionnaires unilingues, dans la langue de départ et la langue d'arrivée. En permettant de procéder à ces recoupements et à des vérifications d'une langue à l'autre, la combinaison d'ouvrages bilingues et unilingues garantit la qualité et la justesse des équivalences établies. Il existe de bons ouvrages lexicographiques dans bien des domaines de spécialité, mais ils sont souvent loin d'être complets et à jour et ils ne tiennent pas toujours compte des synonymes et des variantes lexicales. Le traducteur sera fréquemment appelé à établir lui-même l'équivalence des termes à partir de documents unilingues.

Si nous reprenons l'exemple du rapport annuel, l'étape consacrée à la recherche documentaire devrait aboutir, sur le plan notionnel, à la sélection d'ouvrages paralexicographiques de base tels que Comment lire les états financiers publié par l'Institut ca- 
nadien des valeurs mobilières, dans lequel le profane trouvera, pour la partie comptable, une explication claire des quatre états financiers contenus dans un rapport annuel au Canada. À cela, il conviendra d'ajouter, pour la partie descriptive, un ou deux exemplaires de rapports annuels rédigés en français dans le même domaine de spécialisation que l'original anglais.

Pour ce qui est des sources lexicographiques, un ouvrage comme le Dictionnaire de la comptabilité et des disciplines connexes de Fernand Sylvain offre toutes les garanties terminologiques voulues. L'étudiant y trouvera non seulement les équivalences anglais-français, mais aussi une définition, des renvois synonymiques, des variantes géographiques. Et comme il s'agit d'un livre très complet et fiable, il y a peu de chances que le recours à d'autres dictionnaires soit requis. Toutefois, la consultation de Terminology for Accountants publié par l'Institut canadien des comptables agréés, de Terminologie comptable de l'Ordre des comptables agréés du Québec et de l'un ou l'autre dictionnaire de comptabilité européen peut aussi s'avérer très intéressante et utile. Enfin, il y aura lieu de se procurer la documentation lexicographique pertinente à la sphère d'activité de l'entreprise elle-même.

Après avoir rassemblé une documentation lexicographique et paralexicographique de base, l'étudiant s'initiera au sujet en lisant les ouvrages paralexicographiques sélectionnés. Cette initiation, très rudimentaire, doit permettre à l'étudiant de comprendre l'essence du texte à traduire. C'est la raison pour laquelle le choix des ouvrages de base est capital. Un ouvrage qui n'éclairerait pas le traducteur sur le sujet n'aurait aucune valeur documentaire.

L'étape suivante du processus traductionnel porte sur l'analyse détaillée du texte de départ, en vue d'en comprendre tout le contenu. Cette analyse comporte une importante dimension terminologique puisqu'il s'agit de déterminer avec précision et exactitude le sens du texte et donc des unités terminologiques qui en sont un des éléments constituants. De nature sémasiologique, c'est-à-dire allant du terme à la notion, la recherche terminologique à ce niveau visera à cerner les notions inconnues ou imparfaitement maîtrisées. La détermination du sens se fait généralement dans la langue de départ, à l'aide d'ouvrages lexicographiques unilingues, mais elle peut aussi se faire en langue d'arrivée lorsqu'une documentation bilingue fiable existe. C'est le cas, par exemple, du Dictionnaire de la comptabilité et des disciplines connexes de Fernand Sylvain, dans lequel le traducteur traduisant de l'anglais au français peut aisément, grâce aux définitions, s'approprier les notions en langue d'arrivée. Si les ouvrages lexicographiques ne permettent pas d'établir avec certitude le sens d'un terme, le recours à des documents paralexicographiques en langue de départ s'imposera. Même si la recherche du sens constitue l'activité essentielle de l'étape d'analyse, elle s'accompagne souvent d'une première opération d'établissement d'équivalences ou de transfert lexical. Tel est le cas lorsque des documents bilingues sont consultés, l'équivalent y étant fourni d'office. Il arrive aussi que la recherche de l'équivalent facilite la compréhension de l'unité terminologique et de la notion qu'elle recouvre.

La compréhension du texte étant acquise, l'étape du transfert ou de la traduction proprement dite peut commencer. L'activité terminologique du processus de transfert consiste en l'établissement d'équivalences. Ces équivalences sont de deux types : terminologiques et phraséologiques. Dans un premier temps, il s'agit de trouver la traduction en langue d'arrivée des termes ou unités terminologiques repérés en langue de départ et de s'assurer de l'exactitude des équivalences proposées ou établies. Dans un deuxième temps, comme un terme figure généralement en contexte et qu'une bonne traduction suppose une équivalence de discours, il s'agit d'intégrer l'équivalent dans son contexte en tenant compte de l'idiomaticité de la langue de spécialité concernée. Ainsi, il ne suffit 
pas de connaître l'équivalent terminologique de notes et de consolidated financial statements dans le titre de la rubrique Notes to Consolidated Financial Statements d'un rapport annuel, il faut aussi savoir que notes to se traduit dans ce contexte par notes afférentes à, et cette équivalence relève du domaine phraséologique.

Trois sources de documentation sont utiles à ce stade-ci du travail : 1) les ouvrages lexicographiques bilingues et unilingues, 2) les ouvrages paralexicographiques et, dans cette catégorie, 3) les documents parallèles en langue d'arrivée, quand ils existent.

Les ouvrages lexicographiques serviront principalement à l'établissement des équivalences. En général, la consultation des dictionnaires, lexiques et vocabulaires bilingues permettent de trouver l'équivalent recherché. Toutefois, lorsque les données fournies par les ouvrages bilingues sont incomplètes ou douteuses, le recours aux ouvrages unilingues, lexicographiques et paralexicographiques, s'impose, et la recherche terminologique à laquelle le traducteur doit alors procéder est dite onomasiologique, c'està-dire partant de la notion vers le terme. Nous pouvons utilement appliquer au traducteur la méthode de recherche d'équivalents proposée au terminologue dans $M e ́-$ thodologie de la recherche terminologique ponctuelle ${ }^{10}$ :

En consultant des dictionnaires, des lexiques, des vocabulaires bilingues, le terminologue [le traducteur] arrive le plus souvent à trouver l'attestation de l'équivalence entre deux termes. Par exemple, le Dictionnaire de la comptabilité atteste l'équivalence des termes $b a$ lance sheet et bilan : le terminologue [le traducteur] y trouve la confirmation que ces termes recouvrent la même notion. Ce dictionnaire étant jugé comme une source fiable, le terminologue [le traducteur] pourra immédiatement communiquer le résultat de sa recherche à l'usager [utiliser le résultat de sa recherche]. (...)

Lorsque l'équivalence entre ces termes de langues différentes ne peut être établie clairement à l'aide d'ouvrages bilingues fiables, le terminologue [le traducteur] après avoir exploité les sources multilingues s'il y a lieu, se tourne vers des sources unilingues. En s'inspirant des informations qu'il a recueillies dans les sources de la langue de départ et, s'il y a lieu, lors de l'analyse des éléments de la demande [du texte], le terminologue [le traducteur] cherche dans les sources de la langue d'arrivée le terme qui désigne la réalité exprimée par le terme de la langue de départ. Le terminologue [le traducteur] n'ayant pu se servir du terme de la langue de départ pour amorcer la recherche, il se retrouve alors en situation de recherche unilingue orientée de la notion au terme. Lorsqu'il croit avoir trouvé ce terme après avoir fait appel aux étapes pertinentes de recherche, il compare les données qu'il a recueillies à son sujet à celles qu'il avait rassemblées à propos du terme de la langue de départ, afin de vérifier s'il y a bien équivalence entre les termes.

La lecture et la consultation de documents parallèles, c'est-à-dire de documents en langue d'arrivée comparables à ceux de la langue de départ, permettront de vérifier si l'usage sanctionne réellement les équivalents terminologiques établis, de trouver les équivalents phraséologiques et de respecter l'idiomaticité de discours propre au domaine de spécialisation en cause. Les documents parallèles sont une source documentaire fréquemment sous-utilisée. Les raisons le plus souvent invoquées pour justifier le non-recours à cette source vont du manque de temps à la non-accessibilité des ouvrages. L'intérêt de ces ouvrages est pourtant évident puisqu'ils offrent un modèle à suivre et fournissent souvent des solutions toutes faites sur le plan de la phraséologie spécialisée. En tant qu'outils d'apprentissage, ils sont d'une grande utilité pour l'étudiant. C'est ainsi que la consultation d'un rapport annuel rédigé en français permettra à l'étudiant de trouver, par exemple, la traduction quasi consacrée du texte de l'Auditors' Report (Rapport des vérificateurs) qui figure dans tout rapport annuel. Il lui suffira de recopier les passages identiques ou de s'inspirer du texte parallèle pour produire une traduction conforme à l'usage dans ce domaine. 
Le déroulement chronologique de la recherche des sens et de la recherche des équivalents fait régulièrement l'objet d'interventions de la part des praticiens. Certains préconisent la recherche du sens de toutes les unités terminologiques du texte avant de procéder à la recherche des équivalents. D'autres sont d'avis que la recherche du sens et la recherche de l'équivalent doivent être effectuées simultanément pour chaque unité terminologique. Les opinions sont partagées et il s'agit là beaucoup plus d'une question d'organisation et de préférence personnelles que de principe méthodologique, l'essentiel étant de bien comprendre le sens de l'unité avant de la traduire. Par ailleurs, le traducteur se demande souvent s'il a intérêt à effectuer toute la recherche terminologique (sens et équivalence) avant d'aborder la traduction du texte. Doit-il avoir relevé toutes les unités terminologiques du texte de départ et en avoir trouvé les équivalents avant de trańsposer le texte en langue d'arrivée ? Il s'agit ici encore d'une décision personnelle et individuelle. Certains traducteurs aiment suivre ce déroulement tandis que d'autres préfèrent effectuer la recherche terminologique à mesure qu'ils progressent dans le texte. Une solution intermédiaire, très répandue parmi les praticiens, consiste à travailler sur quelques unités terminologiques à la fois. La longueur du texte, le degré de lexicalisation des unités terminologiques, la disponibilité des sources documentaires sont autant de facteurs qui justifient le choix de l'une ou l'autre démarche.

En résumé, si nous mettons en parallèle le processus traductionnel et la recherche documentaire et terminologique, le travail du traducteur pourrait suivre la progression proposée dans le schéma ci-dessous. L'activité documentaire et terminologique y intervient essentiellement pendant les trois premières étapes du processus traductionnel et a pour rôle d'aider à élucider le texte à traduire et de fournir les équivalents en langue d'arrivée. De plus, une certaine activité de recherche phraséologique peut encore survenir pendant l'étape de la restructuration afin de s'assurer que le texte traduit est le plus conforme possible à l'usage ayant cours dans le domaine de spécialisation concerné. Toutefois, si l'étape du transfert a été faite consciencieusement et avec rigueur, les trois dernières étapes n'impliquent plus de recherche terminologique ou documentaire proprement dite.

Pour conclure, nous aimerions soumettre à la réflexion quelques considérations d'ordre pédagogique. Comme nous avons tenté de le démontrer, l'enseignement de la traduction spécialisée présuppose l'acquisition d'une méthode de recherche documentaire et terminologique. Cette acquisition doit se faire de façon autonome et individuelle et la longueur de l'apprentissage va varier d'un étudiant à l'autre en fonction des compétences et aptitudes de chacun. Une certaine souplesse est donc requise dans l'organisation du cours pour permettre à chaque étudiant d'analyser et d'évaluer régulièrement avec le professeur l'efficacité de la démarche suivie et pour permettre au professeur de faire profiter l'ensemble des étudiants des expériences de chacun. Mais, si l'acquisition d'une méthode de travail et de recherche constitue l'infrastructure d'un cours de traduction spécialisée, elle n'en est pourtant pas l'objectif. La méthode de travail est un moyen et non une fin en soi. Un cours de traduction spécialisée doit avoir pour objectif final la production d'un texte de qualité dans le domaine de spécialisation choisi, et c'est ce texte qui doit servir de base à l'évaluation du travail de l'étudiant. Même si, pédagogiquement, il est utile et nécessaire d'apprécier l'effort fourni et les progrès réalisés en cours de formation, l'évaluation finale doit porter sur le produit fini, en l'occurrence un texte traduit. La compétence documentaire et terminologique ne doit donc en aucun cas supplanter, dans le processus d'évaluation, la compétence traductionnelle de l'étudiant, car un texte bien documenté n'est pas nécessairement un texte bien traduit. 


\begin{tabular}{lc}
\hline $\begin{array}{l}\text { Processus } \\
\text { traductionnel }\end{array}$ & Activité \\
& documentaire/terminologique \\
\hline
\end{tabular}

Prise de connaissance

et lecture globale

du texte à traduire

Détermination du domaine

Sélection de la documentation pertinente

Lecture d'initiation au sujet/domaine

Analyse/compréhension du texte

Transfert de la langue de départ à la langue d'arrivée

\section{Restructuration}

Recherche ponctuelle de nature sémasiologique

Recherche d'équivalents terminologiques et phraséologiques

(Recherche d'équivalents phraséologiques)

\section{Relecture}

\section{Révision}

Notes

1. Les programmes de traduction qui ne comportent pas de cours de traduction spécialisée offrent à la place deux ou trois cours de traduction portant sur un domaine précis de spécialisation, tels que la traduction commerciale, la traduction juridique, la traduction médicale, etc.

2. Parmi les définitions peu éclairantes, on trouve, par exemple, "la traduction de textes non techniques ".

3. Certains cabinets ou services de traduction classent les textes en généraux, semi-techniques ou techniques ou, tout simplement, d'après la spécialité. Quant aux théoriciens de la traduction, ils proposent divers classements, fondés sur des critères fonctionnels, situationnels ou autres.

4. L'initiation au Vocabulaire général d'orientation scientifique (V.G.O.S.) et sa maîtrise constituerait une excellente base lexicale pour l'apprenti traducteur puisque les mots qui le composent sont communs à un grand nombre de spécialités.

5. Pour une définition, voir R. Galisson et D. Coste (1976) : Dictionnaire de didactique des langues, Paris, Hachette, à l'article spécialité.

6. Tina Célestin et al. (1984) : Méthodologie de la recherche terminologique ponctuelle, Québec, gouvernement du Québec, pp. 17-18.

7. Rachel Boutin-Quesnel et al. (1979) : Vocabulaire systématique de la terminologie, Québec, Éditeur officiel, p. 33.

8. Pour une description détaillée des étapes du processus traductionnel, voir Jean-Paul Bénard et Paul Horguelin (1979) : Pratique de la traduction, Montréal, Linguatech, pp. 17-53. 
9. Cette initiation est indispensable au traducteur et devrait être acquise avant d'aborder la traduction spécialisée.

10. Tina Célestin et al., op. cit., pp. 59-60.

\section{BIBLIOGRAPHIE}

BÉNARD, Jean-Paul et Paul HORGUELIN (1979) : Pratique de la traduction. Version générale, $2^{e}$ éd., Montréal, Linguatech.

BOUTIN-QUESNEL, Rachel et al. (1978) : Vocabulaire systématique de la terminologie, Québec, Éditeur officiel.

CÉLESTIN, Tina, Gilles GODBOUT et Pierrette VACHON-L'HEUREUX (1984) : Méthodologie de la recherche terminologique ponctuelle, Québec, gouvernement du Québec.

DUBUC, Robert (1980) : Manuel pratique de terminologie, $2^{\mathrm{e}}$ éd., Paris, CILF.

GALISSON, R. et D. COSTE (1976) : Dictionnaire de didactique des langues, Paris, Hachette

INSTITUT CANADIEN DES COMPTABLES AGRÉÉS (1976) : Terminology for Accountants, Toronto.

INSTITUT CANADIEN DES VALEURS MOBILIËRES (1980) : Comment lire les états financiers, Montréal.

ORDRE DES COMPTABLES AGRÉÉS DU QUÉBEC (1966-1982) : Terminologie comptable, Montréal. RONDEAU, Guy (1983) : Introduction à la terminologie, $2^{e}$ éd., Montréal, Gaëtan Morin.

SYLVAIN, Fernand (1982) : Dictionnaire de la comptabilité et des disciplines connexes, $2^{\mathrm{e}}$ éd. revue et augmentée, Toronto, Institut canadien des comptables agréés. 